\title{
New insights into the basic biology of acute graft-versus-host-disease
}

\author{
Alicia Li, ${ }^{1}$ Ciril Abraham, ${ }^{1}$ Ying Wang ${ }^{1,2}$ and Yi Zhang ${ }^{1,2}$
}

${ }^{1}$ Fels Institute for Cancer Research \& Molecular Biology and ${ }^{2}$ Department of Microbiology and Immunology, Lewis Katz School of Medicine, Temple University, Philadelphia, PA, USA

Haematologica 2020

Volume 105(11):2540-2549

\section{Correspondence:}

YI ZHANG

yi.zhang@temple.edu

Received: April 30, 2020.

Accepted: July 20, 2020.

Pre-published: August 13, 2020.

doi:10.3324/haematol.2019.240291

(C)2020 Ferrata Storti Foundation

Material published in Haematologica is covered by copyright. All rights are reserved to the Ferrata Storti Foundation. Use of published material is allowed under the following terms and conditions:

https://creativecommons.org/licenses/by-nc/4.0/legalcode. Copies of published material are allowed for personal or internal use. Sharing published material for non-commercial purposes is subject to the following conditions:

https://creativecommons.org/licenses/by-nc/4.0/legalcode, sect. 3. Reproducing and sharing published material for commercial purposes is not allowed without permission in writing from the publisher.

\section{ABSTRACT}

A lthough allogeneic hematopoietic stem cell transplantation is an important therapy for many hematologic and non-hematologic diseases, acute graft-versus-host disease $(\mathrm{aGvHD})$ is a major obstacle to its success. The pathogenesis of aGvHD is divided into three distinct phases which occur largely as the result of interactions between infused donor $\mathrm{T}$ cells and numerous cell types of both hematopoietic and non-hematopoietic origin. In light of the disease's immensely complex biology, epigenetics has emerged as a framework with which to examine aGvHD. This review focuses on new findings that clarify the roles that specific epigenetic regulators play in T-cell-mediated aGvHD development and discusses how their modulation could disrupt that process with beneficial effects. DNA methyltransferases, histone methyltransferases and histone deacetylases are the most closely studied regulators across a GvHD priming, induction and effector phases and have been manipulated using drugs and other methods in both murine models and clinical trials, with varying degrees of success. Antigen-presenting cells, effector $T$ cells and memory $T$ cells, among others, are targeted and affected by these regulators in different ways. Finally, our review highlights new directions for study and potential novel targets for modulation to abrogate aGvHD.

\section{Introduction}

The success of allogeneic hematopoietic stem cell transplantation (allo-HSCT) is significantly hampered by acute graft-versus-host disease ( $\mathrm{aGvHD}$ ), which is caused by donor $\mathrm{T}$ cells that recognize and react to histocompatibility differences between the donor and host. It occurs in sequential priming, induction and effector phases (Figure 1). ${ }^{1}$ During priming, preparative irradiation and chemotherapeutic regimens for allo-HSCT can damage the patient's tissues, leading to release of damage-associated molecular patterns (DAMP) and pathogen-associated molecular patterns (PAMP), as well as activation of host antigen-presenting cells (APC) such as dendritic cells. ${ }^{1-6}$ Activated APC, including hematopoietic and non-hematopoietic cells, upregulate antigen-presenting molecules and costimulatory molecules to prime transplanted donor $\mathrm{T}$ cells. ${ }^{1-7}$ During induction, $\mathrm{T}$-cell receptors on donor $\mathrm{T}$ cells react to alloantigens presented by host APC and undergo robust proliferation and differentiation into effector $\mathrm{T}$ cells that produce pro-inflammatory cytokines such as tumor necrosis factor (TNF)- $\alpha$, interferon (IFN)- $\gamma$ and interleukin (IL)-17. ${ }^{1}$ Upon persistent exposure to host alloantigens, most of these effector cells ( $~ 90 \%)$ undergo apoptotic contraction, but a proportion survive and become memory T cells. ${ }^{8-10}$ The final effector phase is characterized by infiltration of alloreactive effector cells into aGvHD target organs. ${ }^{1}$ Tissues already damaged by preparative treatments produce chemokines, recruiting $T$ cells to their vicinity, ${ }^{1,11}$ The effector $T$ cells recognize and react to host alloantigens, mediating host tissue injury. The damaged host tissues recruit more alloreactive $\mathrm{T}$ cells and other types of inflammatory cells (e.g., monocytes/macrophages and granulocytes), leading to feed-forward amplification and continuation of aGvHD (Figure 1). ${ }^{1,2,4}$ Chronic GvHD may arise following or independently of aGvHD, but due to the conditions' differing pathogeneses and clinical manifestations, chronic GvHD will not be discussed in this review.

Because aGvHD is T-cell-mediated, significant progress has been made in understanding how alloreactive T cells are induced and sustained. APC may be primed 


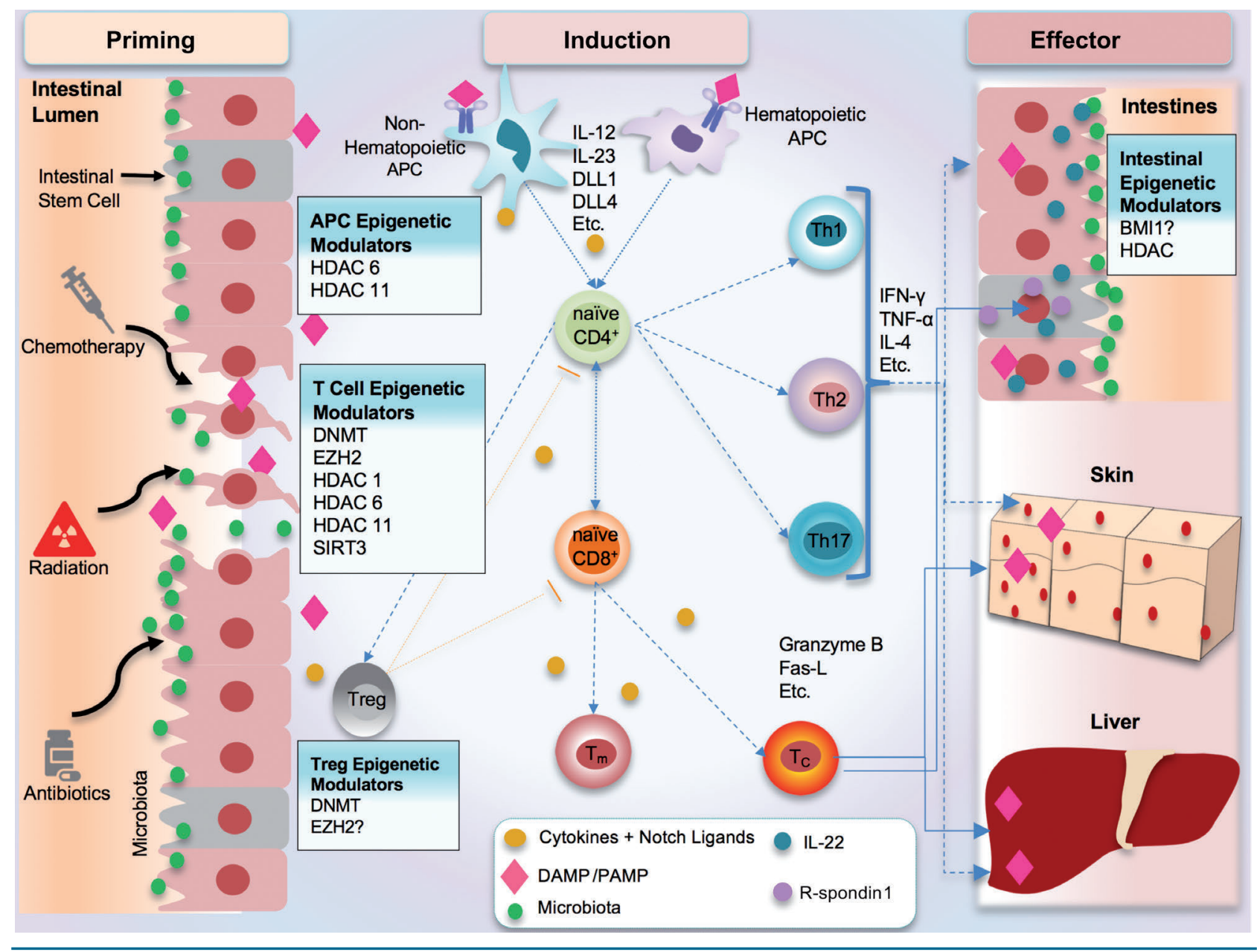

Figure 1. Role of epigenetic regulators in the development of acute graft-versus-host-disease. Acute graft-versus-host disease (aGvHD) develops through three sequential phases: priming, induction and effector. In some cases, following prophylactic treatment and conditioning, the integrity of the intestinal epithelium becomes compromised and leads to the release of damage-associated molecular patterns (DAMP) and pathogen-associated molecular patterns (PAMP). These molecules result in the activation of hematopoietic and non-hematopoietic antigen-presenting cells (APC). Subsequent APC interactions lead to the activation, differentiation and proliferation of T cells. The different subsets of T cells play numerous roles in the pathogenesis of aGvHD. Th1, Th2, Th17, and the cytotoxic T cells interact with target organs to promote tissue damage. In the intestines, intestinal stem cells are notably damaged, impairing tissue regeneration capabilities, contributing to the feed-forward cascade of aGvHD. Epigenetic regulators play a role in each of the three phases allowing for the possibility of therapeutic interventions. HDAC: histone deacetylase; IL: interleukin; DLL: delta-like; IFN: interferon; TNF: tumor necrosis factor; DNMT: DNA methyltransferases

to produce special cytokines (e.g., IL-12, IL-23) and Notch ligands (e.g., Delta-like 1 and 4; DLL1 and DLL4) which instruct antigen-activated $\mathrm{T}$ cells to differentiate into distinct lineages of GvHD-mediating effector $\mathrm{T}$ cells., ${ }^{5,12-15}$ Other groups have reviewed these topics elegantly, so we focus on a related area of investigation: understanding how extracellular stimuli are converted to gene programs that promote or abrogate alloreactive T-cell development and responses, and leveraging them to reduce aGvHD.

Epigenetic modifications are one such mechanism. Epigenetics refers to heritable molecular determinants of phenotype that are independent of DNA sequence. Major contributors include DNA methylation on cytosine nucleotides, histone modification and chromatin structure. Proteins governing these modifications have loosely been termed epigenetic regulators. ${ }^{16}$ This review will discuss advances in our understanding of epigenetic regulation, either by direct effects or via interactions with other molecules, of alloreactive $\mathrm{T}$-cell responses and these responses' roles in a GvHD; we identify the roles that specific regulators play and interventions targeting these reg- ulators for aGvHD prevention and treatment (Table 1). We also acknowledge the contributions of non-hematopoietic cells to the development of aGvHD, whether via their own function or their impact on T cells.

\section{Epigenetic effects on and sensitization of antigen-presenting cells}

To allow for proper engraftment, allo-HSCT patients may undergo conditioning regimens before donor $\mathrm{T}$ cells are infused. Consequently, DAMP from injured cells, PAMP from gut bacteria and pro-inflammatory cytokines are released, priming APC. ${ }^{1}$ In the setting of murine alloHSCT, non-hematopoietic APC, alongside professional hematopoietic APC, are also known to prime alloreactive T cells. ${ }^{2,6,17}$ Upon activation following tissue damage, APC upregulate major histocompatibility complex class II and costimulatory molecules (e.g., CD40, CD80, CD86) and secrete cytokines (e.g., IL-4, IL-12, IL-23, DLL1, DLL4), 
Table 1. Preclinical studies of acute graft-versus-host disease investigating epigenetic mechanisms.

\begin{tabular}{lll} 
Enzyme & \multicolumn{1}{c}{ Cells } & Key findings \\
EZH2 & CD8 ${ }^{+} / \mathrm{CD} 4^{+}$T cells & Ezh2 KO impairs proliferation, differentiation and expansion; it reduces aGvHD but preserves \\
& & GvL. ${ }^{28}$ Ezh2 inhibition with DZNep inhibits ongoing GrHD but preserves the GvL effect. ${ }^{30}$
\end{tabular}

CD8 ${ }^{+} / \mathrm{CD} 4^{+} \mathrm{T}$ cells $\quad$ Administration of the EZH2 inhibitor GSK126, which specifically reduces H3K27me3 without affecting the protein, failed to prevent aGvHD in mice. In contrast, targeting T-cell EZH2 protein by inhibiting HSP90 reduced aGvHD in mice undergoing allo-HSCT. ${ }^{33}$

CD8 ${ }^{+} \mathrm{T}$ cells $\quad$ EZH2 controls $\mathrm{CD} 8{ }^{+} \mathrm{T}$ memory precursor formation and antitumor activity.

\begin{tabular}{|c|c|c|}
\hline DNMT & $\begin{array}{l}\mathrm{CD} 8^{+} / \mathrm{CD} 4^{+} \text {T cells } \\
\text { Treg }\end{array}$ & $\begin{array}{l}\text { Inhibition impairs activation, expansion, and secretion of cytokines. }{ }^{43} \\
\text { Inhibition by Aza increased Treg frequency through hypomethylation of Foxp3. }{ }^{42,43,67}\end{array}$ \\
\hline HDAC (Pan) & $\mathrm{CD}^{+} / \mathrm{CD} 4^{+} \mathrm{T}$ cells & $\begin{array}{l}\text { Pan-inhibition using SAHA results in reduced proliferative and cytotoxic activity of } \\
\text { anti-CD3 activated T cells. }{ }^{34}\end{array}$ \\
\hline HDAC6 & CD8 ${ }^{+} \mathrm{T}$ cells & Inhibition of HDAC6 impairs CD8 ${ }^{+}$T-cell proliferation and function in a GvHD-like model..$^{38}$ \\
\hline HDAC11 & $\mathrm{CD}^{+} / \mathrm{CD}^{+} \mathrm{T}$ cells & $\begin{array}{l}\text { KO of Hdacll increased T-cell proliferation rates and effector function resulting in } \\
\text { more rapid and potent aGvHD. }{ }^{36}\end{array}$ \\
\hline SIRT3 & $\mathrm{CD}^{+} / \mathrm{CD}^{+} \mathrm{T}$ cells & $\begin{array}{l}\text { Loss of Sirt3 results in decreased aGvHD severity due to decreased activation and } \\
\text { production of ROS while maintaining GvT.40 }\end{array}$ \\
\hline HDAC (Pan) & $\mathrm{APC}$ & $\begin{array}{l}\text { Pan-inhibition of HDAC with SAHA reduced aGvHD, resulting in a drastic decrease in } \\
\text { pro-inflammatory cytokine expression and induced high level expression of IDO to suppress } \\
\text { alloreactive T cells. }{ }^{20,23}\end{array}$ \\
\hline HDAC (Pan) & IEC & $\begin{array}{l}\text { Butyrate treatment reduced GvHD severity, improved IEC junction integrity and reduced } \\
\text { IEC apoptosis. In addition, the decrease in } \mathrm{H} 4 \text { acetylation, butyrate transporter and receptor } \\
\text { levels due to allo-HSCT inflammation were reversed. }{ }^{90}\end{array}$ \\
\hline
\end{tabular}

shaping T-cell responses., ${ }^{5,12-15}$ Immunosuppressive molecules such as IL-10, indoleamine-2,3-dioxygenase (IDO) and programmed death ligand 1 (PD-L1) may be upregulated to repress alloreactive $\mathrm{T}$-cell responses, shifting them to become tolerogenic. ${ }^{18-21}$ Epigenetic regulators convert these signals into the aforementioned markers and molecules.

\section{Histone deacetylases' multiple functions in the sensitization of hematopoietic antigen-presenting cells \\ Two classes of enzyme regulate histone acetylation sta-} tus: histone acetyltransferases (HAT) and histone deacetylases (HDAC). HAT acetylate histone lysine substrates and open compacted chromatin, allowing transcription factors to access DNA. ${ }^{22} \mathrm{HDAC}$ decrease histone lysine tail acetylation, repressing gene transcription. ${ }^{16}$ Epigenetic studies of hematopoietic APC sensitization have primarily focused on the impact of HDAC (Figure 1).

One of the first studies anchoring epigenetics to aGvHD, helmed by Reddy and colleagues, brought to light the role of histone acetylation in aGvHD. ${ }^{23} \mathrm{HDAC}$ are important for APC production of pro-inflammatory cytokines and immunosuppressive molecules. ${ }^{20,23}$ Preclinical studies have shown that in vivo administration of the pan-HDAC inhibitor suberoylanilide hydroxamic acid (SAHA) reduced aGvHD. ${ }^{23}$ SAHA treatment did not impair T-cell responses to host antigens, but significantly decreased the production of inflammatory cytokines, TNF- $\alpha$, IL-1 and IFN- $\gamma$, by APC. Subsequent studies confirmed that treatment with SAHA resulted in a marked decrease in pro-inflammatory cytokines (e.g., TNF- $\alpha$, IL-
12, IL-6) in APC, which are important in promoting alloreactive T-cell responses. ${ }^{20}$ SAHA inhibited IL- 6 production in dendritic cells stimulated by variable toll-like receptor (TLR) agonists (e.g., TLR2, TLR3, TLR4 and TLR9). In lipopolysaccharide-stimulated dendritic cells, SAHA treatment induced high-level expression of IDO to suppress alloreactive T-cell responses. ${ }^{20}$

Villagra et al. highlighted the importance of HDAC11 in repressing the negative regulation that murine APC exerted on T-cell responses. ${ }^{18}$ Using chromatin immunoprecipitation, researchers determined that upon overexpression of HDAC11 in APC, there was decreased acetylation of histone $4(\mathrm{H} 4)$ at the distal $I 110$ promoter. This was associated with decreased IL-10 transcription upon lipopolysaccharide stimulation. In contrast, HDAC11 knockdown using shRNA had the opposite effect, resulting in the induction of IL-10 expression. Accordingly, silencing HDAC11 expression in APC impaired antigenspecific T-cell responses, whereas overexpression of HDAC11 in APC caused tolerant CD4 ${ }^{+} \mathrm{T}$ cells to transition to an immunogenic phenotype..$^{18}$

HDAC6 is a positive regulator of tolerogenic APC. Normally, HDAC6 forms a complex with signal transducer and activator of transcription (STAT) 3 that is recruited to the Il10 promoter. Silencing HDAC6 resulted in decreased STAT3 phosphorylation and reduced IL-10 production. ${ }^{19}$ The opposing effects of HDAC11 and HDAC6 in regulating IL-10, a cytokine that can tip the balance between reactivity and tolerance in dendritic cells, ${ }^{18,19}$ underline the importance of understanding how individual HDAC regulate APC function. More specific HDAC 
inhibitors, rather than pan-HDAC inhibitors, may be appropriate targets for further study.

\section{Sensitization of non-hematopoietic cells}

Emerging evidence indicates the importance of nonhematopoietic cells in aGvHD. ${ }^{17}$ Koyama et al. demonstrated that antigen presentation from non-hematopoietic cells could induce lethal aGvHD independently of T-cell interactions with hematopoietic APC. ${ }^{2}$ Microbiota in the gastrointestinal tract can secrete IL-12 to induce major histocompatibility complex class II upregulation on intestinal epithelial cells (IEC), initiating lethal aGvHD. ${ }^{6}$ In addition, fibroblastic stromal cells in the lymph nodes have been shown to drive aGvHD through the presentation of Delta-like Notch ligands, DLL1 and DLL4 specifically. ${ }^{5}$ Inhibition of the Notch ligands and receptors conferred protection against GvHD in murine models. ${ }^{5}$ However, we have a limited understanding of the epigenetic effects these non-hematopoietic cells have on $\mathrm{aGvHD}$. In the light of these striking findings, this represents an important avenue for future investigation.

\section{Epigenetic control of alloreactive $\mathbf{T}$ cells}

Upon encountering allogeneic host APC, infused donor $T$ cells are activated and undergo robust proliferation and differentiation into effector $\mathrm{T}$ cells (Figure 1), which include IFN- $\gamma$-producing CD4 ${ }^{+}$Th1 cells, IL-4-producing CD4+ Th2 cells, IL-17-producing CD4+ Th17 cells and cytotoxic $\mathrm{CD}^{+}{ }^{+} \mathrm{T}$ cells. ${ }^{1}$ Effector $\mathrm{T}$ cells mediate tissue injury during aGvHD. Alloantigen-sensitized donor $\mathrm{T}$ cells can also become memory $T$ cells that mediate persistent host tissue injury. Over the past two decades, much research has been undertaken to understand the molecular mechanisms that control the generation and maintenance of alloreactive effector and memory $\mathrm{T}$ cells during the induction phase of aGvHD.

\section{Epigenetic programming of effector T-cell responses EZH2}

Enhancer of zeste homolog 2 (EZH2) is a histone methyltransferase that catalyzes histone 3 lysine 27 trimethylation (H3K27me3), which primarily silences genes, ${ }^{24}$ and is a core component of the polycomb repressive complex-2 (PRC2). ${ }^{24}$ Evidence suggests that EZH2 is involved in Th1 and Th2 polarization ${ }^{25}$ as well as the proliferation and differentiation of hematopoietic stem cells. ${ }^{26}$ EZH2 is also involved in cancer development and progression, ${ }^{24}$ which has stimulated efforts to develop methods of inhibiting the enzyme.

EZH2 plays an essential role in T-cell immune responses. Studies by our group ${ }^{27-30}$ and others ${ }^{31}$ have demonstrated the functional relevance of EZH2 in regulating antigendriven T-cell responses. Using experimental murine models, we discovered EZH2's role in regulating allogeneic Tcell proliferation, differentiation and function..$^{27,28}$ Conditional loss of Ezh2 in donor T cells inhibited aGvHD in mice. Although EZH2-deficient T cells could be activated and underwent initial proliferation, their ability to undergo continual proliferation and expansion became defective during the later stage of $\mathrm{aGvHD}$ induction. ${ }^{28}$ Unexpectedly, as a gene silencer, EZH2 was required to promote the expression of transcription factors T-bet and STAT4, which are critical for effector differentiation. ${ }^{27}$
Subsequent studies revealed that EZH2 regulation of transcription factor expression and function depends on the differentiation stage of antigen-driven $\mathrm{T}$ cells. ${ }^{32}$ Ezh2 knockout in $\mathrm{T}$ cells impaired their differentiation into IFN$\gamma$-producing effector cells. ${ }^{28}$ However, Ezh2 ablation, EZH2 protein inhibition and EZH2 protein destabilization all did not affect graft-versus-leukemia activity, leading to improved overall survival in recipients. ${ }^{28,30,33}$ Thus, targeting EZH2 may represent an effective therapeutic strategy for a GvHD prevention and treatment.

\section{HDAC1, HDAC6 and HDAC11}

HDAC are important for regulating the proliferative and cytotoxic capabilities of activated $T$ cells. Pharmacological inhibition of HDAC by SAHA has been shown to suppress T-cell-receptor-mediated T-cell proliferation through the induction of apoptosis. ${ }^{34}$ Hdac1 knockout in a murine allergic asthma model showed a significant increase in airway inflammation and Th2 cytokine production. ${ }^{35}$ Upon Hdac1 deletion, in vitro studies noted an enhanced induction of Th1 and Th2 cells. ${ }^{35}$ Thus, HDAC1 plays a negative regulatory role for the functions of Th1 and Th2 subsets.

In T cells, HDAC11 may suppress the graft-versus-host reaction. Hdac11 knockout resulted in increased T-cell proliferation and release of pro-inflammatory cytokines associated with upregulation of eomesodermin (EOMES) and T-bet which are important in effector differentiation. ${ }^{36}$ Indeed, decreased expression of HDAC11 exacerbated aGvHD in mice. ${ }^{36}$

HDAC6 can deacetylate non-histone proteins such as heat shock protein 90 (HSP90)..$^{37}$ Acetylation disrupts HSP90's chaperone function and inhibits LCK phosphorylation. ${ }^{38}$ In a GvHD-like model involving OT-I T-cell transplants to K14-mnOVA mice, control mice developed mucosal and skin lesions, while inhibition of HDAC6 using a specific inhibitor, ACY-1215, prevented similar lesions from forming for 14 days after transplantation. This protective effect was accompanied by dramatically decreased production of $\mathrm{CD}^{+}$effector $\mathrm{T}$ cells that secreted high levels of IL-2 and IFN $-\gamma \cdot{ }^{38}$ Further studies of these HDAC should be conducted in GvHD models to definitively validate their roles in driving or mitigating aGvHD.

\section{SIRT3}

SIRT3 is a mitochondrial HDAC that regulates metabolic enzyme acetylation. ${ }^{39}$ SIRT3 is expressed in metabolically stressed cells such as alloreactive T cells. ${ }^{40}$ Loss of SIRT3 in donor T cells led to decreased GvHD severity in mice. The protective effect associated with Sirt3 deletion was associated with a reduction in reactive oxygen species and decreased activation and expression of chemokine receptor CXCR3. ${ }^{40}$

\section{DNMT}

Because DNA methyltransferases (DNMT) - DNMT1, DNMT3A, DNMT3B and DNMT3L - can enact global transcription suppression, they have been widely studied in the context of immunity. DNMT1 is the principal enzyme that maintains methylation across DNA replication. ${ }^{41}$ DNMT3A and DNMT3B contribute to methylation maintenance and are also responsible for de novo DNA methylation. ${ }^{41}$ DNMT inhibitors such as 5-azacytidine (Aza) have been shown to impair T-cell activation, expansion and cytokine release early in culture via downregula- 
tion of cell cycle- and cytokine-related genes. ${ }^{42}$ SánchezAbarca et al. treated mice undergoing allo-HSCT with Aza and found that early treatment prevented aGvHD development without increasing regulatory $\mathrm{T}$ cells (Treg); researchers speculated that this was likely due to Aza inhibition of T-cell expansion, which had been demonstrated in vitro. ${ }^{42}$ In a humanized murine allo-HSCT, xenogeneic GvHD model, Aza treatment was also noted to decrease the frequency of IFN- $\gamma$-secreting $\mathrm{CD}^{+}$human $\mathrm{T}$ cells and granzyme $\mathrm{B}$ - and perforin 1 -secreting $\mathrm{CD}^{+}$human $\mathrm{T}$ cells in vivo. ${ }^{43}$ Using Dnmt3 a conditional knockout mice, a recent study by Youngblood et al. revealed the importance of DNMT3A in the regulation of T-cell exhaustion. ${ }^{44}$ Moving forward, similar genetic approaches will be useful in understanding the precise mechanisms of the effect of DNMT inhibition on aGvHD.

\section{Epigenetic programming of alloreactive memory T cells}

A hallmark of aGvHD is cytopathic injury mediated by persistent alloreactive effector $\mathrm{T}$ cells, which can occur within weeks and persist for years after transplantation. ${ }^{1,-9,29}$ Data from our ${ }^{8,9,29}$ studies suggest that memory $T$ cells that develop during aGvHD sustain alloreactive effector cells. , $9,29^{2}$ These alloantigen-sensitized memory $\mathrm{T}$ cells differ from naturally-occurring $T$ cells because the ability of memory $\mathrm{T}$ cells to mediate aGvHD is limited by their $\mathrm{T}$-cell receptor repertoires. ${ }^{45}$ Memory $\mathrm{T}$ cells are generated during the primary immune response from proliferating $\mathrm{T}$ cells upon APC activation. ${ }^{46}$ After reencountering antigens, they undergo rapid and robust proliferation and elaboration of effector function. They have stem cell-like self-renewal properties, are distinguishable from both naive and effector $\mathrm{T}$ cells and are resistant to existing immunosuppressive agents. ${ }^{47,48}$ In fact, whether or not their resilience contributes to the low response rates to current a GvHD therapies $(\sim 40 \%)$ is the subject of ongoing debate. , $^{1,9}$

\section{EZH2}

$\mathrm{EZH} 2$ is required for the development of memory precursors early after antigenic priming, for the maturation of memory $\mathrm{T}$ cells and for the recall response of mature memory $\mathrm{T}$ cells. ${ }^{32}$ EZH2 deficiency in activated $\mathrm{CD}^{+} \mathrm{T}$ cells caused significant skewing toward central memory precursors and drastically increased the relative proportion of terminally differentiated effector cells that were unable to contribute to further expansion. ${ }^{32}$ EZH2 repressed the expression of Blimp-1, ID2 and EOMES, which promote effector differentiation, and promoted and sustained expression of ID3, a gatekeeper critical for memory formation and survival. ${ }^{22}$ Given EZH2's crucial roles in the regulation of alloreactive $\mathrm{T}$ cells, EZH2-mediated memory formation may be responsible for the generation and maintenance of alloreactive memory $\mathrm{T}$ cells during aGvHD.

\section{Other regulators}

Histone methyltransferase SUV39H1 may play a role in repressing memory genes. Upon infection with Listeria monocytogenes, SUV39H1-defective CD8 ${ }^{+} \mathrm{T}$ cells demonstrated enhanced "long-term memory reprogramming," allowing them to persist in mice. ${ }^{49}$ The Mixed-Lineage Leukemia gene encodes histone lysine methyltransferase $2 \mathrm{~A}$ and may be a regulator of memory Th2 cells. ${ }^{50}$ Protein arginine methyltransferase 5 (PRMT5) has also been implicated as a supporter of memory T-cell reactivation; its inhibition suppressed memory Th1 responses in experimental autoimmune encephalitis. ${ }^{51}$ We anticipate continued studies of these epigenetic regulators that consider their relevance to $\mathrm{a} \mathrm{GvHD}$.

\section{Epigenetic regulation of regulatory $\mathbf{T}$ cells}

Treg with $\mathrm{CD}^{+} \mathrm{CD}^{2} 5^{+} \mathrm{FOXP3}{ }^{+}$phenotype can suppress immune responses via cytokine- and contact-dependent mechanisms. ${ }^{1,52}$ Stable Forkhead box P3 (FOXP3) expression has been considered a critical determinant of Treg identity and activity, but some suggest this characterization may be incomplete, pointing to the importance of independent epigenetic alterations. ${ }^{53}$ Natural Treg (nTreg) and induced Tregs (iTreg) are the most closelystudied Treg subsets in aGvHD; nTreg develop within the thymus while iTreg arise from activated $\mathrm{CD}^{+} \mathrm{T}$ cells in the periphery. ${ }^{54}$ Because they can repress $\mathrm{T}$-cell proliferation and survival, Treg have been established as an important cell population in reducing aGvHD. ${ }^{55,56}$ Indeed, infusion of nTreg into allo-HSCT mice abrogated aGvHD lethality, ${ }^{57}$ and $i$ Treg have been shown to suppress aGvHD in allogeneic models. ${ }^{58,59}$ While nTreg use has achieved preliminarily promising results in clinical trials,$^{56}$ the potential of iTreg is less clear. Each has disadvantages; there are few nTreg in the peripheral blood and their use requires ex vivo expansion..$^{0}$ On the other hand, iTreg, with unstable FOXP3 expression, are often unable to maintain a suppressive phenotype..$^{61-63}$

\section{DNMT}

Researchers have attempted to stabilize FOXP3 expression by maintaining demethylation of the Foxp3 locus. ${ }^{64,65}$ As DNMT are involved in maintaining methylation, they are thought to contribute to Foxp3 suppression. Indeed, without demethylation of a CpG island in the Foxp3 locus, cells' FOXP3 expression and suppressive ability are limited. ${ }^{66}$ The use of DNMT inhibitors to sustain Treg stability and abrogate aGvHD has achieved some success.

Choi et al. showed that treatment with the DNMT inhibitors decitabine and Aza induced Treg from CD4 ${ }^{+} \mathrm{CD} 25$ cells. ${ }^{67}$ Transplantation of decitabine- and Aza-treated cells into mice undergoing allo-HSCT reduced clinical aGvHD and improved survival. ${ }^{67}$ Directly treating mice with Aza after allo-HSCT resulted in similar effects. Interestingly, these suppressive effects of iTreg were found to be maintained even in Foxp3 knockout cells, suggesting that their anti-GvHD activity may be downstream or independent of FOXP3 expression. ${ }^{67}$ In a humanized murine allo-HSCT, xenogeneic GvHD model, in vivo Aza treatment was associated with longer survival and lower xenogeneic GvHD scores. ${ }^{43}$ Researchers suggested that Aza treatment induced Il2 promoter hypomethylation, leading to increased IL-2 expression and augmented Treg proliferation. ${ }^{43}$

\section{EZH2}

EZH2 is known to co-localize with FOXP3 ${ }^{68}$ and has also been implicated in the maintenance of Treg identity after activation. ${ }^{69}$ Tumes et al. noted that iTreg differentiation is impaired without EZH2. ${ }^{31}$ Ablation of Ezh2 led to autoimmunity associated with a faulty FOXP3-depen- 
dent gene expression program in activated Treg. ${ }^{69}$ Ezh2 deletion in in vivo murine Treg reprogrammed them to express an effector phenotype that could potentially be unfavorable for aGvHD. ${ }^{70}$ In vitro pharmacological EZH2 inhibition also impaired iTreg differentiation, resulting in a significantly decreased frequency of iTreg..$^{70}$ Human iTreg treated with the same inhibitor were unable to maintain suppressive activity. ${ }^{70}$ However, since inhibition of EZH2 potently suppressed persistence and expansion of effector $\mathrm{T}$ cells, the impact of pharmacological inhibition of EZH2 on Treg is likely context-dependent.

\section{HDAC}

Akimova et al. established that human Treg express a unique combination of HDAC compared to effector $\mathrm{T}$ cells, and that treatment with several different HDAC inhibitors augmented the suppressive function of Treg in vitro. ${ }^{71}$ Specific HDAC have been implicated in modulating Treg function, including HDAC3 (for both nTreg and iTreg), ${ }^{72}$ HDAC9 and HDAC6. Inhibition of HDAC9 had a positive effect on FOXP3 expression and nTreg generation. $^{73}$ Deletion of Hdacb or Sirt1 resulted in similar increases in FOXP3 expression and augmented nTreg function. ${ }^{74}$ Interestingly, combined pharmacological inhibition of HDAC6 and SIRT1 had a synergistic effect on increasing $n$ Treg function in vivo in mice. It is likely that the two enzymes share mechanisms in their effect via the deacetylation of FOXP3. ${ }^{74}$ However, because these studies were not conducted in the context of GvHD, their results should be taken as an indication for GvHD study in the future.

In the gut, butyrate and other short-chain fatty acids (SCFA) produced by commensal bacteria may also assist with the induction and maintenance of Treg in the periphery. ${ }^{75,76}$ Possessing more Lachnospiraceae- and Ruminococcaceae-family bacteria, which belong to the class Clostridia, was correlated with greater H3 acetylation, a greater Treg/Th17 cell ratio and greater protection against aGvHD. ${ }^{77}$ Members of the Clostridia class produce SCFA that comprise colonocytes' primary energy source. $^{78}$ The SCFA butyrate has notable HDAC inhibitory activity. ${ }^{79}$ Butyrate delivery via drinking water increased peripheral Treg in mice treated with broadspectrum antibiotics. ${ }^{75}$ Notably, this increase did not occur in mice deficient in the conserved non-coding sequence (CNS) 1 enhancer, which is part of the Foxp3 locus. Treg isolated from these mice exhibited improved suppressor function in vitro compared to antibiotic-treated mice that did not receive butyrate. Treating $\mathrm{CD}^{+} \mathrm{T}$ cells with butyrate during non-specific activation in vitro was also able to induce Treg, so researchers examined the effect of the treatment on Foxp3 locus deacetylation. Butyrate-treated naive $\mathrm{CD}^{+} \mathrm{FOXP3}-\mathrm{T}$ cells that were non-specifically activated for 3 days showed significant increases in Foxp3 promoter and CNS 1, 2 and 3 acetylation at $\mathrm{H} 3 \mathrm{~K} 27 .^{75}$

Furusawa et al. noted that feeding mice with butyrylated high-amylose maize starches significantly increased differentiation of colonic Treg; these Treg were able to suppress chronic intestinal inflammation brought on by adoptive transfer of $\mathrm{CD}^{+} \mathrm{CD} 45 \mathrm{RB}^{\text {hi }}$ cells into $\mathrm{Rag}^{1^{-/}}$ mice. ${ }^{76}$ Through chromatin immunoprecipitation analysis, researchers verified that butyrate treatment increased global acetylation levels, but also acetylation at histone $\mathrm{H} 3$ at (i) the Foxp3 promotor region and CNS3 prior to
FOXP3 induction and (ii) CNS1 over the course of Treg differentiation. ${ }^{76}$ Though not directly related to aGvHD, these are important findings pertaining to gut inflammation that should provide direction for further study.

\section{Other regulators}

Endothelial cell dysfunction, specifically the loss of endothelial cell-derived thrombomodulin, has been associated with steroid-refractory aGvHD..$^{80}$ Ranjan et al. showed that thrombomodulin is essential for the generation of protease-activated protein C; incubation of human $\mathrm{T}$ cells with activated protein $\mathrm{C}$ prior to their transplantation into humanized mice increased Treg frequency and improved xenogeneic GvHD compared to non-incubated human $\mathrm{T}$ cells. ${ }^{80}$ This activity was speculated to take place via an epigenetic pathway that has yet to be investigated.

\section{Epigenetic programs that influence tissue injury and regeneration during acute graft-versus-host disease}

The effector phase is characterized by migration and infiltration of alloreactive effector cells into a GvHD target organs and cytotoxic attack (Figure 1). Areas surrounding tissue commonly affected by aGvHD produce chemokines (e.g., CXCL9 and CXCL10) that recruit effector $\mathrm{T}$ cells. ${ }^{1,11}$ The cells recognize major histocompatibility complex and/or minor histocompatibility antigen mismatches and attack tissue via a cytotoxic response mediated by cell-surface factors and cytokines. Alloreactive effector $\mathrm{T}$ cells attack tissue through mechanisms that include Fas-Fas ligand interactions, perforin- and granzyme-mediated killing and TNF- $\alpha$ induction of cell death. ${ }^{1}$

Concurrently, tissue regeneration from both cytotoxic damage and potentially pre-allo-HSCT conditioning commences. Intestinal stem cells (ISC) are crucial for the regeneration of the intestinal epithelium after injury. However, ISC are also a target of effector T cells during aGvHD, causing the intestinal epithelium to be trapped in a cycle of repeated damage. Interestingly, IL-22 plays a central role in protecting the intestinal epithelium and ISC. ${ }^{81,82}$ During aGvHD, IL-23-responsive intestinal lymphoid cells produce and secrete IL-22. However, intestinal lymphoid cells are also targeted and eliminated during disease progression, leading to IL-22 deficiency and further ISC damage. ${ }^{81}$ Regeneration can be boosted through Wnt pathway stimulation using the Wnt agonist RSpondin1.83 Treatment with R-Spondin1 before alloHSCT expanded ISC and treatment after transplant enhanced surviving ISC proliferation, allowing for fortification of the intestinal lumen and aGvHD inhibition. ${ }^{83}$

Little is known about epigenetic regulation of the Wnt pathway-dependent and IL-22-mediated regeneration processes in the context of GvHD. Recent studies have suggested that BMI1, a polycomb repressive complex-1 (PRC1) component important for hematopoietic stem cell renewal, is expressed in the ISC and progenitor compartments. ${ }^{84}$ PRC1 is known to enact its function in gene silencing via recognition of $\mathrm{H} 3 \mathrm{~K} 27 \mathrm{me} 3 .{ }^{85}$ Bmil knockout resulted in reduced ISC proliferation and significant increases in cell cycle regulators $\mathrm{p} 16^{\mathrm{INK4a}}$ and $\mathrm{p} 19^{\mathrm{ARF} 84} \cdot{ }^{84}$ 
BMI1 contributes to ISC self-renewal, which is co-regulated by the Wnt pathway and Notch. The interaction between BMI1 and the Wnt pathway in regulating stem cell self-renewal has been validated in a separate study. ${ }^{86}$

Yeste et al. noted that STAT3 regulates $I 122$ promoter accessibility. ${ }^{87}$ When STAT3-deficient $\mathrm{CD}^{+} \mathrm{T}$ cells were activated in the presence of IL-21, which normally induces 1122 transcription, IL-22 production was significantly decreased. STAT3-deficient cells also showed decreases in $\mathrm{H} 3$ and $\mathrm{H} 4$ acetylation, decreases in $\mathrm{H} 3 \mathrm{~K} 4 \mathrm{me} 3$ and increases in H3K9me3 and H3K27me3 at the 1122 promoter. This could involve a number of epigenetic regulators, including histone methyltransferases (e.g., G9A, SUV39H1, EZH2) and enzymes that modify histone acetylation. ${ }^{87}$

Recent studies have also implicated the microbiome in aGvHD development and exacerbation. The inflammatory conditions associated with aGvHD, as well as pre-transplant preparatory regimens, can harm commensal bacteria populations and compromise the normal functioning of gastrointestinal cells, which in turn result in more severe aGvHD. ${ }^{88-90}$ For instance, aGvHD inflammation was associated with a loss of SCFA-producing bacteria in the Clostridiales order in both humans and mice. ${ }^{89}$ Mathewson et al. showed that the levels of the HDAC inhibitor butyrate in IEC were significantly decreased after exposure to allo-HSCT inflammation; this led to decreased histone $\mathrm{H} 4$ acetylation and decreased expression of the butyrate transporter and receptor, SLC5A8 and GPR43, respectively, in IEC. ${ }^{90}$ Increasing intragastric butyrate levels restored $\mathrm{H} 4$ acetylation, decreased GvHD severity and improved IEC junction integrity..$^{90}$ Butyrate treatment was also associated with significantly less IEC apoptosis; among other effects, treatment led to lower expression of pro-apoptotic proteins, higher expression of the anti-apoptotic protein BCL2 and higher expression of junctional proteins occludin and $\mathrm{JAM}^{.0}$ The promoter regions of $B \mathrm{cl} 220$ (encoding BCL-2) and Fllr (encoding JAM) were noted to be directly associated with $\mathrm{H} 4$ acetylation. ${ }^{90}$ Though the study did not discuss these changes' impact on recipient physiology, it is plausible that increased IEC junction integrity and decreased apoptosis prevented immune cell infiltration and PAMP/DAMP from escaping the gut, reducing the severity of the aGvHD.

Deeper examination of this aspect of aGvHD biology and its associated pathways is crucial because it represents a less commonly pursued paradigm in a GvHD treatment: fostering recovery of damaged tissues (Figure 1). ${ }^{91}$

\section{Pharmacological modulation of acute graft-versus-host disease by targeting epigenetic pathways}

Development of epigenetic therapy is a particularly active area of cancer research because of such therapies' potential to selectively target chromatin-modifying enzyme-mediated disease mechanisms. ${ }^{16}$ Epigenetic therapy may produce fewer adverse effects than conventional cytotoxic chemotherapies and may influence response to immunotherapy in various cancers. This logic applies to the search for drugs which modify epigenetic mechanisms controlling alloreactive $\mathrm{T}$-cell responses to reduce aGvHD while preserving graft-versus-leukemia activity (Table 2).

\section{HDAC inhibitors}

In two clinical trials, patients receiving related and unrelated donor HSCT were treated with the pan-HDAC inhibitor vorinostat (SAHA) after myeloablative conditioning to determine the drug's efficacy at preventing aGvHD. Clinical trials showed a cumulative incidence of grade II-IV aGvHD of $22 \%$ by day $100 . .^{92,93}$ Correlative tests on vorinostat-treated patients' blood samples showed a significant reduction in IL- 6 . $^{93}$ Overall, treatment with vorinostat was deemed a safe and efficacious strategy for preventing GvHD. Treatment with the panHDAC inhibitor panobinostat, in conjunction with corticosteroids, was also recently investigated for the mitigation of ongoing GvHD. ${ }^{94}$ Treatment had an approximately $40 \%$ response rate, and these responses were noted across grades II and III GvHD in different organ systems. The results are inconclusive because the trial lacked sufficient power, but are nevertheless promising. ${ }^{94}$

\section{DNMT inhibitors}

Clinically, Aza and decitabine have been used in the context of allo-HSCT for the express purpose of reducing the disease burden before transplantation and as maintenance

Table 2. Selected clinical trials of epigenetic inhibitors in acute graft-versus-host disease.

\begin{tabular}{ll} 
Drug & Main Conclusion \\
Vorinostat (SAHA) & $\begin{array}{l}\text { HDAC inhibition with vorinostat in combination with standard prophylaxis resulted in reduced incidence } \\
\text { of severe aGvHD. Phase I/II trial. } .^{92}\end{array}$ \\
& HDAC inhibition with vorinostat was safe and efficacious in unrelated donor allo-HSCT patients receiving \\
& myeloablative conditioning and methotrexate. Results showed a low cumulative incidence of severe aGvHD. \\
& Phase II trial. ${ }^{93}$ \\
Panobinostat & HDAC inhibition with panobinostat in addition to glucocorticoids as primary therapy for aGvHD was deemed safe. \\
& However, the study did not have sufficient power to address efficacy. Phase I/II trial. ${ }^{94}$ \\
\hline 5-Azacytidine & $\begin{array}{l}\text { DMNT inhibition by Aza after donor lymphocyte infusion as salvage therapy was well tolerated and no patients } \\
\text { developed grade III-IV aGvHD. Phase I trial. }{ }^{95}\end{array}$
\end{tabular}

DMNT inhibition by Aza after allogenic stem cell transplantation increased circulating Treg in patients. Phase I/II trials. ${ }^{96}$

SAHA: suberoylanilide hydroxamic acid; HDAC: histone deacetylase; aGVHD: acute graft-versus-host-disease; allo-HSCT: allogeneic hematopoietic stem cell transplantation; Aza: 5-azacytidine; DNMT: DNA methyltransferase; Treg: regulatory T cell 
and salvage therapy. ${ }^{99}$ In studies using Aza or decitabine treatment in the setting of blood cancers or myelodysplastic syndromes to reduce disease burden before transplantation, there were no significant findings with regard to aGvHD. ${ }^{98,99}$ DNMT inhibitor treatment after allo-HSCT has typically been a component of salvage or maintenance therapy and has had some success in mitigating aGvHD. Ghobadi et al. treated patients with Aza after donor lymphocyte infusion; no patients developed severe aGvHD (III-IV) and there was no aGvHD-caused mortality. ${ }^{95}$ Similarly, Schroeder et al. provided Aza treatment alongside donor lymphocyte infusion upon patients' relapse and saw a 3.2-fold increase in Treg and a 1.9-fold increase in Treg frequency after four cycles of Aza treatment in patients who relapsed early after allo-HSCT. ${ }^{96}$ Goodyear et al. found that although the incidence of $\mathrm{aGvHD}$ was lower in treatment groups than in control groups, Treg increases in post-transplant acute myeloid leukemia patients were only observed within the first 3 months of treatment. ${ }^{97}$ These results suggest that early treatment may be required for a beneficial effect on aGvHD.

Because of its comparative success, it may be fruitful for future clinical trials to expand on the post-transplant, early HDAC inhibitor treatment paradigm. Of note, DNMT inhibitors were not typically used for a GvHD prevention, so patients often received other treatments (e.g., methotrexate) which were not standardized across studies.

\section{EZH2 inhibitors}

In vivo administration of GSK126 failed to reduce $\mathrm{aGvHD}$ and did not affect the development of alloreactive effector T cells in preclinical studies. ${ }^{33}$ This is in contrast to observations that EZH2 deficiency led to aGvHD blockade in various murine allo-HSCT models. ${ }^{28}$ The mechanism of action of EZH2 in mediating aGvHD induction is therefore likely independent of its canonical target H3K27me3. ${ }^{33}$ Notably, EZH2 protein depletion by DZNep led to arrest of ongoing GvHD in experimental mice, ${ }^{30}$ indicating that targeting EZH2 may lead to new strategies to treat ongoing GvHD.

An interaction between HSP90 and EZH2 has also been shown to be vital for the stability and function of EZH2. ${ }^{33}$ A lack of HSP90 marks EZH2 for ubiquitination via the proteasome. Treatment of activated T cells with the HSP90 inhibitor AUY922 significantly decreased EZH2 protein levels while leaving histone methylation intact. HSP90 inhibitor treatment significantly decreased alloreactive $\mathrm{T}$ cell responses and aGvHD in mice, affirming EZH2's involvement in aGvHD pathogenesis and the non-canonical hypothesis. ${ }^{33}$
The Food and Drug Administration has approved the EZH2 inhibitor tazemetostat specifically for the treatment of epithelioid sarcoma. We anticipate that this inhibitor may be used to target alloreactive memory $\mathrm{T}$ cells to reduce aGvHD in the future.

\section{Future directions}

As the epigenetics of aGvHD biology is a young area of study, there is much room for further investigation, both in elucidating mechanisms surrounding the action of known enzymes and in exploring the roles of new regulators documented here and beyond. Nevertheless, enormous progress has been made through the identification of critical enzymes and mechanisms. Next steps will be to further map how their pathways intersect amid the multitude of cell types and interactions that comprise aGvHD. Some epigenetic regulators (e.g., EZH2 and HDAC6) have points of commonality in their mechanisms of action (via HSP90). ${ }^{19,33,37}$ Advances will illuminate these locations of confluence such that more effective, integrated therapies may be developed. Additionally, a single regulator (e.g., HDAC11) may have beneficial or detrimental effects at different stages of cell development; understanding these situations will be vital for treatment. Also bringing promise for epigenetic intervention are those aspects of aGvHD pathogenesis that are T-cellindependent, such as microbiome injury.

Different tissues, hematopoietic and non-hematopoietic, have distinct roles in mediating aGvHD immunopathology. Further investigation of the epigenetics surrounding the role of non-hematopoietic APC would likely be beneficial for the field. In addition, tissueintrinsic mechanisms that contribute to inhibition of aGvHD have been somewhat overlooked. These include those controlling tissue regeneration, ${ }^{81-83}$ and those modulating tissue-resident APC, which are critical for local aGvHD induction. ${ }^{100}$ Furthermore, aGvHD blocks peripheral tolerance of host-reactive $\mathrm{T}$ cells by elimination of lymph node fibroblastic reticular cells that induce T-cell tolerance in the gut. ${ }^{101}$ Thus, future studies should also investigate the epigenetic mechanisms that regulate tissue regeneration and regulation of the graft-versus-host reaction, as suggested by Reddy and colleagues. ${ }^{91}$

\section{Acknowledgments}

This study was supported by grants from the NCI (CA172106-01, YZ), NHLBI (HL127351-01A1, YZ) and NIAID (AI143256-01A1, YZ).

\section{References}

1. Zeiser R, Blazar BR. Acute graft-versus-host disease - biologic process, prevention, and therapy. N Engl J Med. 2017;377(22):21672179.

2. Koyama M, Kuns RD, Olver SD, et al. Recipient nonhematopoietic antigen-presenting cells are sufficient to induce lethal acute graft-versus-host disease. Nat Med. 2011;18(1):135-142.

3. Shlomchik WD, Couzens MS, Tang CB, et al. Prevention of graft versus host disease by inactivation of host antigen-presenting cells.
Science. 1999;285(5426):412-415.

4. Zhang Y, Louboutin JP, Zhu J, Rivera AJ, Emerson SG. Preterminal host dendritic cells in irradiated mice prime CD8 + T cell-mediated acute graft-versus-host disease. J Clin Invest. 2002;109(10):1335-1344

5. Chung J, Ebens CL, Perkey E, et al. Fibroblastic niches prime $T$ cell alloimmunity through Delta-like Notch ligands. J Clin Invest. 2017;127(4):1574-1588.

6. Koyama M, Mukhopadhyay P, Schuster IS et al. MHC class II antigen presentation by the intestinal epithelium initiates graft-versus-host disease and is influenced by the microbiota. Immunity. 2019;51(5):885- 898.e7.

7. Wu CJ, Ritz J. Induction of tumor immunity following allogeneic stem cell transplantation. Adv Immunol. 2006;90:133-173.

8. Zhang Y, Joe G, Hexner E, Zhu J, Emerson SG. Host-reactive CD8+ memory stem cells in graft-versus-host disease. Nat Med. 2005;11(12):1299-1305

9. Zhang Y, Joe G, Hexner E, Zhu J, Emerson SG. Alloreactive memory $T$ cells are responsible for the persistence of graft-versus-host disease. J Immunol. 2005;174(5):3051-3058.

10. Kaech SM, Wherry EJ, Ahmed R. Effector and memory T-cell differentiation: implications for vaccine development. Nat Rev 
Immunol. 2002;2(4):251-262.

11. Reshef R, Luger SM, Hexner EO, et al. Blockade of lymphocyte chemotaxis in visceral graft-versus-host disease. N Engl J Med. 2012;367(2):135-145.

12. Palucka K, Banchereau J. Dendritic-cellbased therapeutic cancer vaccines. Immunity. 2013;39(1):38-48.

13. Sandy AR, Chung J, Toubai T, et al. T cellspecific notch inhibition blocks graft-versushost disease by inducing a hyporesponsive program in alloreactive CD4+ and CD8+ T cells. J Immunol. 2013;190(11):5818-5828.

14. Amsen D, Antov A, Flavell RA. The different faces of Notch in T-helper-cell differentiation. Nat Rev Immunol. 2009:9(2):116-124.

15. Tran IT, Sandy AR, Carulli AJ, et al. Blockade of individual Notch ligands and receptors controls graft-versus-host disease. J Clin Invest. 2013;123(4):1590-1604.

16. Baylin SB, Jones PA. Epigenetic Determinants of Cancer. Cold Spring Harb Perspect Biol. 2016;8(9):a019505.

17. Jones SC, Murphy GF, Friedman TM, Korngold R. Importance of minor histocompatibility antigen expression by nonhematopoietic tissues in a CD4+ $\mathrm{T}$ cellmediated graft-versus-host disease model. J Clin Invest. 2003;112(12):1880-1886.

18. Villagra A, Cheng F, Wang HW, et al. The histone deacetylase HDAC11 regulates the expression of interleukin 10 and immune tolerance. Nat Immunol. 2009;10 (1):92-100.

19. Cheng F, Lienlaf M, Wang HW, et al. A novel role for histone deacetylase 6 in the regulation of the tolerogenic STAT3/IL-10 pathway in APCs. J Immunol. 2014;193(6):28502862.

20. Reddy P, Sun Y, Toubai T, et al. Histone deacetylase inhibition modulates indoleamine 2,3-dioxygenase-dependent DC functions and regulates experimental graft-versus-host disease in mice. J Clin Invest. 2008;118(7):2562-2573

21. Ni X, Song Q, Cassady K, et al. PD-L1 interacts with CD80 to regulate graft-versusleukemia activity of donor CD8+ T cells. J Clin Invest. 2017;127(5):1960-1977.

22. Lee DY, Hayes JJ, Pruss D, Wolffe AP. A positive role for histone acetylation in transcription factor access to nucleosomal DNA. Cell. 1993;72(1):73-84

23. Reddy P, Maeda Y, Hotary K, et al. Histone deacetylase inhibitor suberoylanilide hydroxamic acid reduces acute graft-versushost disease and preserves graft-versusleukemia effect. Proc Natl Acad Sci U S A. 2004;101(11):3921-3926.

24. Margueron R, Reinberg D. The Polycomb complex PRC2 and its mark in life. Nature. 2011;469(7330):343-349.

25. Jacob E, Hod-Dvorai R, Ben-Mordechai OL, Boyko Y, Avni O. Dual function of polycomb group proteins in differentiated murine T helper (CD4+) cells. J Mol Signal. 2011;6:5

26. Kamminga LM, Bystrykh LV, de Boer A, et al. The Polycomb group gene Ezh2 prevents hematopoietic stem cell exhaustion. Blood. 2006;107(5):2170-2179

27. Tong $\mathrm{Q}$, He S, Xie F, et al. Ezh2 Regulates transcriptional and posttranslational expression of T-bet and promotes Th1 cell responses mediating aplastic anemia in mice. J Immunol. 2014;192(11):5012-5022.

28. He S, Xie F, Liu Y, et al. The histone methyltransferase Ezh2 is a crucial epigenetic regulator of allogeneic $T$ cell responses mediating graft-versus-host disease. Blood. 2013;122 (25):4119-4128
29. Kato K, Cui S, Kuick R, et al. Identification of stem cell transcriptional programs normally expressed in embryonic and neural stem cells in alloreactive CD8+ $\mathrm{T}$ cells mediating graft-versus-host disease. Biol Blood Marrow Transplant. 2010;16(6):751 771.

30. He S, Wang J, Kato K, et al. Inhibition of histone methylation arrests ongoing graft-versus-host disease in mice by selectively inducing apoptosis of alloreactive effector T cells. Blood. 2012;119(5):1274-1282

31. Tumes DJ, Onodera A, Suzuki A, et al. The polycomb protein Ezh2 regulates differentiation and plasticity of CD4(+) T helper type 1 and type 2 cells. Immunity. 2013;39(5): 819-832.

32. He S, Liu Y, Meng L, et al. Ezh2 phosphorylation state determines its capacity to maintain CD8(+) T memory precursors for antitumor immunity. Nat Commun. 2017;8(1) 2125

33. Huang Q, He S, Tian Y, et al. Hsp90 inhibition destabilizes Ezh2 protein in alloreactive $T$ cells and reduces graft-versus-host disease in mice. Blood. 2017;129(20):2737-2748.

34. Li N, Zhao D, Kirschbaum M, et al. HDAC inhibitor reduces cytokine storm and facilitates induction of chimerism that reverses lupus in anti-CD3 conditioning regimen. Proc Natl Acad Sci U S A. 2008;105(12): 4796-4801.

35. Grausenburger R, Bilic I, Boucheron N, et al. Conditional deletion of histone deacetylase 1 in T cells leads to enhanced airway inflammation and increased Th2 cytokine production. J Immunol. 2010;185(6):3489-3497.

36. Woods DM, Woan KV, Cheng F, et al. T cells lacking HDAC11 have increased effector functions and mediate enhanced alloreactivity in a murine model. Blood. 2017;130(2): 146-155.

37. de Zoeten EF, Wang L, Butler K, et al. Histone deacetylase 6 and heat shock protein 90 control the functions of Foxp3(+) Tregulatory cells. Mol Cell Biol. 2011;31(10): 2066-2078.

38. Tsuji G, Okiyama N, Villarroel VA, Katz SI Histone deacetylase 6 inhibition impairs effector CD8 T-cell functions during skin inflammation. J Allergy Clin Immunol. 2015;135(5):1228-1239.

39. Hirschey MD, Shimazu T, Goetzman E, et al. SIRT3 regulates mitochondrial fatty-acid oxidation by reversible enzyme deacetylation. Nature. 2010;464(7285):121-125.

40. Toubai T, Tamaki H, Peltier DC, et al Mitochondrial deacetylase SIRT3 plays an important role in donor $\mathrm{T}$ cell responses after experimental allogeneic hematopoietic Transplantation. J Immunol. 2018;201(11): 3443-3455.

41. Bestor TH. The DNA methyltransferases of mammals. Hum Mol Genet. 2000;9(16) 2395-2402

42. Sanchez-Abarca LI, Gutierrez-Cosio S, Santamaria $C$, et al. Immunomodulatory effect of 5-azacytidine (5-azaC): potential role in the transplantation setting. Blood. 2010;115(1):107-121.

43. Ehx G, Fransolet G, de Leval L, et al. Azacytidine prevents experimental xenogeneic graft-versus-host disease without abrogating graft-versus-leukemia effects. Oncoimmunology. 2017;6(5):e1314425.

44. Youngblood B, Hale JS, Kissick HT, et al Effector CD8 T cells dedifferentiate into long-lived memory cells. Nature. 2017;552 (7685):404-409.

45. Anderson BE, McNiff J, Yan J, et al. Memory $\mathrm{CD} 4+\mathrm{T}$ cells do not induce graft-versus- host disease. J Clin Invest. 2003;112(1):101108

46. Masopust D, Kaech SM, Wherry EJ, Ahmed $\mathrm{R}$. The role of programming in memory $\mathrm{T}$ cell development. Curr Opin Immunol. 2004;16(2):217-225

47. Wu Z, Bensinger SJ, Zhang J, et al Homeostatic proliferation is a barrier to transplantation tolerance. Nat Med. 2004;10(1):87-92

48. Maltzman JS, Turka LA. T-cell costimulatory blockade in organ transplantation. Cold Spring Harbor Perspect Med. 2013;3(12): a015537.

49. Pace L, Goudot C, Zueva E, et al. The epigenetic control of stemness in CD8(+) T cell fate commitment. Science. 2018;359(6372): 177-186.

50. Yamashita M, Hirahara K, Shinnakasu R, et al. Crucial role of MLL for the maintenance of memory $\mathrm{T}$ helper type 2 cell responses. Immunity. 2006:24(5):611-622.

51. Webb LM, Amici SA, Jablonski KA, et al. PRMT5-selective inhibitors suppress inflammatory $\mathrm{T}$ cell responses and experimental autoimmune encephalomyelitis. J Immunol. 2017;198(4):1439-1451.

52. Negrin RS. Graft-versus-host disease versus graft-versus-leukemia. Hematology. 2015; 2015(1):225-230.

53. Ohkura N, Hamaguchi M, Morikawa $\mathrm{H}$, et al. T cell receptor stimulation-induced epigenetic changes and Foxp3 expression are independent and complementary events required for Treg cell development. Immunity. 2012;37(5):785-799.

54. Workman CI, Szymczak-Workman AI Collison LW, Pillai MR, Vignali DA. The development and function of regulatory $\mathrm{T}$ cells. Cell Mol Life Sci. 2009;66(16):26032622

55. Cohen JL, Trenado A, Vasey D, Klatzmann D, Salomon BL. CD4(+)CD25(+) immunoregulatory $T$ cells: new therapeutics for graft-versus-host disease. J Exp Med. 2002;196(3):401-406.

56. Di Ianni M, Falzetti F, Carotti A, et al. Tregs prevent GVHD and promote immune reconstitution in HLA-haploidentical transplantation. Blood. 2011;117(14):3921-3928.

57. Taylor PA, Lees CJ, Blazar BR. The infusion of ex vivo activated and expanded CD4(+)CD25(+) immune regulatory cells inhibits graft-versus-host disease lethality. Blood. 2002;99(10):3493-3499.

58. Hippen KL, Merkel SC, Schirm DK, et al. Generation and large-scale expansion of human inducible regulatory $T$ cells that suppress graft-versus-host disease. Am J Transplant. 2011;11(6):1148-1157.

59. Li J, Heinrichs J, Haarberg K, et al. HYSpecific induced regulatory $\mathrm{T}$ cells display high specificity and efficacy in the prevention of acute graft-versus-host disease. Immunol. 2015;195(2):717-725.

60. Tang Q, Henriksen KJ, Bi M, et al. In vitroexpanded antigen-specific regulatory $\mathrm{T}$ cells suppress autoimmune diabetes. J Exp Med. 2004;199(11):1455-1465.

61. Zhou X, Bailey-Bucktrout SL, Jeker LT, et al. Instability of the transcription factor Foxp3 leads to the generation of pathogenic memory $T$ cells in vivo. Nat Immunol. 2009;10(9):1000-1007.

62. Koenecke C, Czeloth N, Bubke A, et al Alloantigen-specific de novo-induced Foxp3+ Treg revert in vivo and do not protect from experimental GVHD. Eur J Immunol. 2009:39(11):3091-3096.

63. Beres A, Komorowski R, Mihara M, Drobyski WR. Instability of Foxp3 expres- 
sion limits the ability of induced regulatory $T$ cells to mitigate graft versus host disease. Clin Cancer Res. 2011;17(12):3969-3983.

64. Polansky JK, Kretschmer K, Freyer J, et al. DNA methylation controls Foxp3 gene expression. Eur J Immunol. 2008;38(6):16541663.

65. Zheng Y, Josefowicz S, Chaudhry A, Peng XP, Forbush K, Rudensky AY. Role of conserved non-coding DNA elements in the Foxp3 gene in regulatory T-cell fate. Nature. 2010;463(7282):808-812.

66. Floess S, Freyer J, Siewert C, et al. Epigenetic control of the foxp3 locus in regulatory $\mathrm{T}$ cells. PLoS Biol. 2007:5(2):e38.

67. Choi J, Ritchey J, Prior JL, et al. In vivo administration of hypomethylating agents mitigate graft-versus-host disease without sacrificing graft-versus-leukemia. Blood. 2010;116(1):129-139.

68. Arvey A, van der Veeken J, Samstein RM, Feng Y, Stamatoyannopoulos JA, Rudensky AY. Inflammation-induced repression of chromatin bound by the transcription factor Foxp3 in regulatory $T$ cells. Nat Immunol. 2014;15(6):580-587.

69. DuPage M, Chopra G, Quiros J, et al. The chromatin-modifying enzyme Ezh2 is critical for the maintenance of regulatory $\mathrm{T}$ cell identity after activation. Immunity. 2015;42(2):227-238.

70. Goswami S, Apostolou I, Zhang J, et al. Modulation of EZH2 expression in T cells improves efficacy of anti-CTLA-4 therapy. J Clin Invest. 2018;128(9):3813-3818.

71. Akimova T, Ge G, Golovina T, et al. Histone/protein deacetylase inhibitors increase suppressive functions of human FOXP3+ Tregs. Clin Immunol. 2010;136(3): 348-363.

72. Wang L, Liu Y, Han R, et al. FOXP3+ regulatory $\mathrm{T}$ cell development and function require histone/protein deacetylase 3. J Clin Invest. 2015;125(3):1111-1123.

73. Tao R, de Zoeten EF, Ozkaynak E, et al. Deacetylase inhibition promotes the generation and function of regulatory $\mathrm{T}$ cells. Nat Med. 2007:13(11):1299-1307.

74. Beier UH, Wang L, Han R, Akimova T, Liu Y, Hancock WW. Histone deacetylases 6 and 9 and sirtuin-1 control Foxp3+ regulatory $\mathrm{T}$ cell function through shared and isoformspecific mechanisms. Sci Signal. 2012;5(229):ra45

75. Arpaia N, Campbell C, Fan X, et al. Metabolites produced by commensal bacteria promote peripheral regulatory T-cell generation. Nature. 2013:504(7480):451-455

76. Furusawa Y, Obata Y, Fukuda S, et al. Commensal microbe-derived butyrate induces the differentiation of colonic regulatory T cells. Nature. 2013;504(7480):446-
450

77. Han L, Jin H, Zhou L, et al. Intestinal Microbiota at engraftment influence acute graft-versus-host disease via the Treg/Th17 balance in allo-HSCT recipients. Front Immunol. 2018;9:669.

78. Lopetuso LR, Scaldaferri F, Petito V, Gasbarrini A. Commensal Clostridia: leading players in the maintenance of gut homeostasis. Gut Pathog. 2013;5(1):23.

79. Waldecker M, Kautenburger T, Daumann H, Busch C, Schrenk D. Inhibition of histonedeacetylase activity by short-chain fatty acids and some polyphenol metabolites formed in the colon. I Nutr Biochem. 2008;19(9):587-593

80. Ranjan S, Goihl A, Kohli S, et al. Activated protein $\mathrm{C}$ protects from GvHD via PAR2/PAR3 signalling in regulatory $\mathrm{T}$-cells. Nat Commun. 2017;8(1):311.

81. Hanash AM, Dudakov JA, Hua G, et al. Interleukin-22 protects intestinal stem cells from immune-mediated tissue damage and regulates sensitivity to graft versus host disease. Immunity. 2012;37(2):339-350.

82. Lindemans CA, Calafiore M, Mertelsmann AM, et al. Interleukin-22 promotes intestinal-stem-cell-mediated epithelial regeneration. Nature. 2015;528(7583):560-564.

83. Takashima S, Kadowaki M, Aoyama K, et al. The Wnt agonist R-spondin1 regulates systemic graft-versus-host disease by protecting intestinal stem cells. J Exp Med. 2011;208(2):285-294

84. Lopez-Arribillaga E, Rodilla V, Pellegrinet L, et al. Bmil regulates murine intestinal stem cell proliferation and self-renewal downstream of Notch. Development. 2015;142(1): 41-50.

85. Sangiorgi E, Capecchi MR. Bmil is expressed in vivo in intestinal stem cells. Nat Genet. 2008;40(7):915-920

86. Cho JH, Dimri M, Dimri GP. A positive feedback loop regulates the expression of polycomb group protein BMI1 via WNT signaling pathway. J Biol Chem. 2013;288(5):3406 3418

87. Yeste A, Mascanfroni ID, Nadeau M, et al IL-21 induces IL-22 production in CD4+ T cells. Nat Commun. 2014;5:3753.

88. Lee SE, Lim JY, Ryu DB, et al. Alteration of the intestinal microbiota by broad-spectrum antibiotic use correlates with the occurrence of intestinal graft-versus-host disease. Biol Blood Marrow Transplant. 2019;25(10): 1933-1943

89. Jenq RR, Ubeda C, Taur Y, et al. Regulation of intestinal inflammation by microbiota following allogeneic bone marrow transplantation. J Exp Med. 2012;209(5):903-911.

90. Mathewson ND, Jenq R, Mathew AV, et al. Gut microbiome-derived metabolites modulate intestinal epithelial cell damage and mitigate graft-versus-host disease. Nat Immunol. 2016;17(5):505-513

91. Wu SR, Reddy P. Tissue tolerance: a distinct concept to control acute GVHD severity. Blood. 2017;129(13):1747-1752.

92. Choi SW, Braun T, Chang L, et al. Vorinostat plus tacrolimus and mycophenolate to prevent graft-versus-host disease after relateddonor reduced-intensity conditioning allogeneic haemopoietic stem-cell transplantation: a phase 1/2 trial. Lancet Oncol. 2014;15(1):87-95

93. Choi SW, Braun T, Henig I, et al. Vorinostat plus tacrolimus/methotrexate to prevent GVHD after myeloablative conditioning, unrelated donor HCT. Blood. 2017;130(15): 1760-1767.

94. Perez L, Fernandez H, Horna P, et al. Phase I trial of histone deacetylase inhibitor panobinostat in addition to glucocorticoids for primary therapy of acute graft-versus-host disease. Bone Marrow Transplant. 2018;53(11) 1434-1444.

95. Ghobadi A, Fiala MA, Abboud CN, et al. A phase I study of azacitidine after donor lymphocyte infusion for relapsed acute myeloid leukemia post allogeneic stem cell transplantation. Blood. 2013;122(21):3320-3320.

96. Schroeder T, Frobel J, Cadeddu RP, et al Salvage therapy with azacitidine increases regulatory $\mathrm{T}$ cells in peripheral blood of patients with AML or MDS and early relapse after allogeneic blood stem cell transplantation. Leukemia. 2013;27(9): 1910-1913

97. Goodyear OC, Dennis M, Jilani NY, et al Azacitidine augments expansion of regulatory $\mathrm{T}$ cells after allogeneic stem cell transplantation in patients with acute myeloid leukemia (AML). Blood. 2012;119(14):3361 3369 .

98. Lübbert M, Bertz $H$, Ruter B et al. Nonintensive treatment with low-dose 5-aza-2'deoxycytidine (DAC) prior to allogeneic blood SCT of older MDS/AML patients. Bone Marrow Transplant. 2009;44(9):585588.

99. Field T, Perkins J, Huang Y, et al. 5-Azacitidine for myelodysplasia before allogeneic hematopoietic cell transplantation. Bone Marrow Transplant. 2010:45(2):255-260.

100. Zhang Y, Shlomchik WD, Joe G, et al. APCs in the liver and spleen recruit activated allogeneic CD8+ T cells to elicit hepatic graftversus-host disease. J Immunol. 2002;169 (12):7111-7118

101. Dertschnig S, Evans P, Santos ESP, et al. Graft-versus-host disease reduces lymph node display of tissue-restricted self-antigens and promotes autoimmunity. J Clin Invest. 2020;130(4):1896-1911. 